Doug Geisler, Eva K. Grebel, and Dante Minniti, eds.

\title{
HST Observations of Young Star Clusters in Interacting Galaxies
}

\author{
Kirk D. Borne \\ Raytheon ITSS and NASA-GSFC, Code 631, Astrophysics Data \\ Facility, Greenbelt, MD 20771 USA \\ William C. Keel \\ University of Alabama, Dept. of Physics and Astronomy, Tuscaloosa, \\ AL 35487-0324 USA \\ Philip N. Appleton \& Curtis Struck \\ Iowa State University, Dept. of Physics and Astronomy, Ames, IA \\ 50011 USA
}

\author{
Ray A. Lucas \& Alfred B. Schultz \\ STScI, 3700 San Martin Drive, Baltimore, MD 21218 USA
}

\begin{abstract}
We present early results from the analysis of HST imaging observations for several pairs of interacting galaxies. We include two cases that were specifically chosen to represent a strong early (young) encounter and a weak late (old) encounter. The goals of the project include a determination of the timing, frequency, strength, and characteristics of the young star clusters formed in these two limiting cases of tidal encounters.
\end{abstract}

\section{Results from the Analysis of HST Imaging Observations}

Multi-band HST imaging data have been obtained for several interacting galaxy systems. These include the Cartwheel Ring Galaxy (Borne et al. 1996; Struck et al. 1996; Appleton et al., in preparation), Arp 81 and Arp 297 (Keel \& Borne, in preparation), and a large sample of ULIRGs (Ultra-Luminous Infrared Galaxies; Borne et al. 2000, and references therein). WFPC2 B-band (F450W) and $I$-band $(\mathrm{F} 814 \mathrm{~W})$ images have been obtained for the Cartwheel, Arp 297, and Arp 81. WFPC2 $I$-band and NICMOS $H$-band (F160W) images have been obtained for the ULIRGs (Borne et al. 1997b, 1999, 2001; Bushouse et al. 2001; Colina et al. 2001). Additional HST archival WFPC2 $V$-band (F606W) images are also available for some of the ULIRGs (Farrah et al. 2001). A wide variety of scientific results from these studies have been published or are in preparation. Here, we report specifically on results pertaining to the young star cluster (YSC) population in these interacting galaxy systems. One of the goals of these investigations is to determine the trigger, timing, frequency, strength, and characteristics of YSC formation in collisions and mergers of gas-rich galaxies. A similar investigation by Gallagher et al. (2001) for Stephan's Quintet reveals 
"several distinct epochs of star formation that appear to trace the complex history of dynamical interactions in this compact group." In a similar manner, we hope to trace the history of star formation in colliding pairs.

It appears certain that the formation of YSCs in interacting galaxies is collision-induced (Whitmore et al. 1993, 1999; Whitmore 2000). A key question is: At what phase of the interaction do the clusters form? We attempt to address this through investigations of a wide variety of galaxy collisions and mergers.

A rich population of $\sim 500$ very blue YSCs is seen in the Cartwheel Ring Galaxy (Appleton et al., in preparation). This galaxy owes its morphology to a direct head-on collision with a companion galaxy. The $B-I$ color image of the Cartwheel shows that the most intensely blue colors emanate from the southwest quadrant of the ring where the bluest and brightest YSCs are detected (Borne et al. 1997a). There are 150 YSCs (i.e., blue clusters) detected in the "spoke" region and in the small inner ring (a secondary by-product of the headon collision), but many more ( 350$)$ YSCs are detected in the primary expanding collision-induced outer ring. We have analyzed the radial color variations in pieshaped sectors around the Cartwheel, averaging azimuthally at a given radius within a sector. The azimuthal average includes the bright clusters plus the galactic background and is generally redder than the clusters, probably because there is some underlying galaxy light which is red and centrally concentrated, with a rough $1 / r$ distribution. The azimuthally averaged radial color distribution and the colors of the YSCs converge to very blue values of $B-I$ at large radius where the YSCs dominate the light of the galaxy (in the outer ring).

While the trigger and timing of YSC formation is clear in the case of the Cartwheel galaxy, there is still some uncertainty as to when the YSCs begin to form in grazing encounters. We are investigating two extremes of the population of such collision configurations: a strong/young encounter (Arp 81) and a weak/old (well developed) encounter (Arp 297). Objects that appear to be YSCs are seen in both cases.

In Arp 297 (NGC 5752/5754), nearly 800 star clusters have been identified, mostly in the larger galaxy (NGC 5754). The latter are much fainter and yet bluer (on average) compared to the clusters in the small companion galaxy (NGC 5752). Thus, this weak/old tidal interaction has stimulated the most luminous clusters to form in the small galaxy, which feels the strongest tidal perturbation, and these clusters probably formed earlier in the interaction (hence their redder colors) compared to the less luminous clusters in NGC 5754, which probably formed much later in the interaction, therefore appearing younger (bluer colors).

The true-color HST image of Arp 81 (NGC 6621/6622) reveals a remarkable pattern of dust lanes and filaments. Many $(\sim 700)$ luminous blue clusters have been identified, clearly demonstrating YSC formation in a young, yet strong, collision. The most populous region for blue clusters in Arp 81 is the contact region between the two galaxies, reminiscent of a similarly intense starburst event in the contact region of the Antennae (NGC 4038/4039; Whitmore et al. 1999). The colors of the YSCs in Arp 81 are intermediate between those of the two galaxies in the Arp 297 galaxy pair (described above), and hence the YSCs in Arp 81 may be of a corresponding intermediate age.

In addition to these results, we find that the ULIRGs show a significant population of very bright star knots or YSCs (Borne et al. 1997b; Surace et 
al. 1998), which are most likely produced during the starburst/merger event. In one case (IRAS 21130-4446), HST's superb angular resolution capability has revealed that this ULIRG is a collision-induced ring galaxy with a "beads on a string" morphology (Fig. 2b; Borne et al. 1997b). These "beads" are probably very bright collision-induced YSCs, similar to (though much brighter than) those seen in the Cartwheel. This ring galaxy may prove to be very useful as a comparative chronometer of the collision age, the ULIRG development age, and the YSC formation age.

Acknowledgments. This work was supported by a Raytheon Sabbatical Research Award and by NASA through HST grants GO-05410.01-93, GO06346.01-95A, and GO-07467.02-96A from the Space Telescope Science Institute, which is operated by AURA, Inc., under NASA contract NAS5-26555.

\section{References}

Borne, K., Appleton, P., Lucas, R. A., Struck, C., \& Schultz, A. B. 1997a, Revista Mexicana de Astronomia y Astrofisica Serie de Conferencias, 6, 141

Borne, K., Bushouse, H., Colina, L., \& Lucas, R. A. 1997b, in Extragalactic Astronomy in the Infrared, ed. G. A. Mamon, T. X. Thuan, \& J. Tran Thanh Van (Paris: Editions Frontieres), 277

Borne, K. D., Bushouse, H., Colina, L., \& Lucas, R. A. 2001, in preparation

Borne, K. D., Bushouse, H., Colina, L., Lucas, R. A., Baker, A., Clements, D., Lawrence, A., Oliver, S., \& Rowan-Robinson, M. 1999, Ap\&SS, 266, 137

Borne, K. D., Bushouse, H., Lucas, R. A., \& Colina, L. 2000, ApJ, 529, L77

Borne, K. D., Lucas, R. A., Appleton, P., Struck, C., Schultz, A. B., \& Spight, L. 1996, in Science with the Hubble Space Telescope - II, ed. P. Benvenuti, F. D. Macchetto, \& E. J. Schreier, 239

Bushouse, H., Borne, K., Colina, L., Lucas, R., Rowan-Robinson, M., Baker, A., Clements, D., Lawrence, A., \& Oliver, S. 2001, ApJ, in press

Colina, L., Borne, K., Bushouse, H., Lucas, R., Rowan-Robinson, M., Lawrence, A., Clements, D., Baker, A., \& Oliver, S. 2001, ApJ, in press

Farrah, D., Rowan-Robinson, M., Oliver, S., Serjeant, S., Borne, K., Lawrence, A., Bushouse, H., Colina, L., \& Lucas, R. 2001, MNRAS, in press

Gallagher, S. C., Charlton, J. C., Hunsberger, S. D., Zaritsky, D., \& Whitmore, B. C. 2001, AJ, in press (astro-ph/0104005)

Surace, J. A., Sanders, D. B., Vacca, W. D., Veilleux, S., \& Mazzarella, J. M. 1998, ApJ, 492, 116

Struck, C., Appleton, P. N., Borne, K. D., \& Lucas, R. A. 1996, AJ, 112, 1868

Whitmore, B. C. 2000, in STScI Symposium Series 14, ed. M. Livio, in press (astro-ph/0012546)

Whitmore, B. C., Schweizer, F., Leitherer, C., Borne, K., \& Robert, C. 1993, AJ, 106, 1354

Whitmore, B. C., Zhang, Q., Leitherer, C., Fall, S. M., Schweizer, F., \& Miller, B. W. 1999, AJ, 118, 1551 